\title{
MS22-04 | The Role of Electrostatic Interactions in IFIT Proteins Complexed With RNA With Different 5' End Predicted by the UBDB+EPMM Method
}

Budniak, Urszula (Department of Chemistry, CNBCh, University of Warsaw, Warsaw, POL); Dominiak, Paulina (Department of Chemistry, CNBCh, University of Warsaw, Warsaw, POL)

My project is focused on IFITs proteins (Interferon-induced proteins with tetratricopeptide repeats) which are expressed in cells infected by viruses. By binding foreign RNA they prevent synthesis of viral proteins in human host cell. IFIT1, IFIT2 and IFIT5 bind different forms of RNA: with triphosphate group or cap at 5' end of RNA. Calculated electrostatic interactions in selected complexes of IFIT1 and IFIT5 proteins with RNA will allow to compare the results with experimental values of binding affinity.

One of the more advanced methods to calculate electrostatic energy is University at Buffalo Pseudoatom DataBank (UBDB) together with Exact Potential Multipole Method (EPMM). UBDB enables reconstruction of charge density for macromolecules in quantitative manner. By UBDB+EPMM approach, which takes also charge penetration effects into account, it is possible to compute electrostatic energies with similar accuracy as with quantum chemistry methods, but much faster. Energy calculations are based on the structures of IFIT5 proteins with pppRNA and IFIT1 with different 5' end of RNA deposited in PDB. I want to verify the hypothesis of the lack of influence of RNA sequence on interaction energy in IFIT-RNA complexes. Moreover, I examine how modifications at 5 ' end of RNA alter interaction strength.

Project is financed from the grant PRELUDIUM11 of National Science Centre, Poland nr 2016/21/N/ST4/03722.

[1] Abbas, Y. M. et al. (2017) PNAS 114(11), E2106-E2115.

[2] Jarzembska, K. N. \& Dominiak, P. M. (2012) Acta Cryst. A68, 139-147.

[3] Volkov A et al. (2004) Chem. Phys. Lett. 391, 170-175. 